Meta

Journal des tradlucteurs

Translators' Journal

\title{
Ambiguïté et traduction
}

\section{Irène V. Spilka}

Volume 26, numéro 4, décembre 1981

URI : https://id.erudit.org/iderudit/004259ar

DOI : https://doi.org/10.7202/004259ar

Aller au sommaire du numéro

Éditeur(s)

Les Presses de l'Université de Montréal

ISSN

0026-0452 (imprimé)

1492-1421 (numérique)

Découvrir la revue

Citer cet article

Spilka, I. V. (1981). Ambiguïté et traduction. Meta, 26(4), 332-337.

https://doi.org/10.7202/004259ar

Ce document est protégé par la loi sur le droit d'auteur. L’utilisation des services d'Érudit (y compris la reproduction) est assujettie à sa politique d'utilisation que vous pouvez consulter en ligne.

https://apropos.erudit.org/fr/usagers/politique-dutilisation/
Cet article est diffusé et préservé par Érudit.

Érudit est un consortium interuniversitaire sans but lucratif composé de l’Université de Montréal, l'Université Laval et l'Université du Québec à Montréal. Il a pour mission la promotion et la valorisation de la recherche. https://www.erudit.org/fr/ 


\section{Ambiguïté et traduction *}

IRÈNE V. SPILKA

Les trois étapes de l'opération traduction - décodage, comparaison, recodage - comportent chacune des difficultés propres, qu'il convient de préciser. Au cours du décodage, le travail du traducteur consiste à prendre connaissance du texte et à comprendre le message qu'il renferme. Si la prise de connaissance ne nécessite guère qu'une compétence linguistique suffisante, la compréhension, par contre, exige davantage, car le message fait référence à la réalité extralinguistique. Pour saisir le contenu et la portée d'un texte, le récepteur doit être en mesure non seulement de le déchiffrer, mais encore de relier le message à la réalité ; autrement dit, il doit posséder une certaine «image du monde" qui coïncide au moins en partie avec celle de l'émetteur, et savoir la projeter sur les mots de l'énoncé afin de leur donner un sens. Le travail du décodage s'effectue donc dans deux plans, celui de la langue et celui de la réalité extralinguistique. En cela, le décodage accompli par le traducteur ne diffère guère de celui que fait l'auditeur ou le lecteur unilingue. Or, les textes sont vagues, car les langues sont sélectives : elles n'obligent pas l'émetteur à formuler toute l'information à transmettre, mais seulement à fournir au récepteur des repères plus ou moins nombreux, dont certains sont obligatoires et d'autres facultatifs, de telle sorte que le récepteur dispose de moyens suffisants pour comprendre ce qui est dit et reconstituer ce qui n'est pas dit. Ainsi l'anglais n'oblige pas le locuteur à préciser le sexe de la personne dont il parle. La phrase (1)

(1) The secretary is absent

ne donne aucune indication quant au sexe de la personne qui assume les fonctions de secrétaire. Cet inconvénient est léger pour le récepteur unilingue, soit que celui-ci traite directement avec son interlocuteur et ait la possibilité de lui demander un complément d'information au besoin, soit qu'il se contente de demeurer dans l'incertitude, à laquelle sa langue l'a habitué sur ce point, l'information manquante n'étant pas pertinente dans toutes les circonstances. Le traducteur, par contre, se voit contraint par les exigences mêmes de sa langue d'arrivée (LA) de préciser ce que la langue de départ (LD) passe volontiers sous silence. Or, il a rarement accès à l'auteur du texte, et il ne peut lever l'ambiguité qu'en interrogeant le contexte (environnement textuel) et la situation (ensemble des conditions extratextuelles entourant l'énoncé), lesquels restent souvent muets. Il semble donc que l'ambiguïté du texte original (TO) et celle de la LD constituent le principal obstacle à surmonter au cours de l'opération de décodage.

Par ambiguïté, nous entendons la propriété qu'ont certains énoncés de recevoir plus d'une interprétation. Par exemple, la phrase

(2) Nagging may be injurious to the health

* Colloque de Glendon, 1980. 
peut signifier

- 'for someone to nag you may be injurious to your health'

- 'for you to nag someone may be injurious to your health'

L'ambiguité de l'exemple (2) est due à la forme linguistique en -ing, qui peut remplir diverses fonctions dans la phrase et donner lieu à des constructions avec antécédent libre. On objectera peut-être que l'intonation, le contexte, ou la situation interviennent pour lever l'ambiguïté, mais cette objection, bien que fondée en théorie, perd de son acuité lorsqu'on sait que, dans la pratique, les textes soumis au traducteur sont souvent fragmentaires et isolés de la situation qui leur a donné lieu, et que, de toute façon, le texte écrit est dépourvu d'indications prosodiques. L'ambiguïté illustrée par l'exemple (2) est donc imputable à la fois à la structure de la phrase anglaise - et donc au système linguistique qui permet d'engendrer de telles phrases -, aux insuffisances de l'écrit par rapport à l'oral, et aux déficiences d'un discours tronqué. Le premier facteur est strictement linguistique, les deux autres relèvent de la communication, en tout cas d'un aspect de la communication qui touche le traducteur.

Nous distinguerons par ailleurs l'ambiguïté linguistique et l'ambiguïté discursive. Dans le premier cas, c'est la similitude des signifiants qui masque la diversité des signifiés; dans le second cas, ce peut être le style communicatif de l'émetteur qui brouille le message, même lorsque l'énoncé demeure entier. L'ambiguiité linguistique peut, en outre, être intralinguistique, c'est-à-dire propre à une langue, ou interlinguistique, c'est-à-dire résultant du rapprochement de deux langues. Enfin, elle peut se situer au niveau lexical, celui des «entrées du dictionnaire», ou au niveau syntaxique, celui des constructions qui articulent la phrase.

Au niveau du lexique, l'homonymie fournit un exemple simple et probant d'ambiguité intralinguistique. Soit

(3) L'officiant tournait le dos au canon

Cela signifie-t-il que l'officiant tournait le dos à une pièce d'artillerie ou au tableau des prières posé sur l'autel? On pourrait multiplier à plaisir les exemples de ce genre, qui sont assez gros pour ne pas présenter de difficulté véritable. Mais l'homonymie peut jouer sur des distinctions plus subtiles. Prenons la phrase suivante, relevée au hasard d'une conversation :

(4) Elaine is a good manager

qui peut signifier

- "Elaine occupies the position of "manager" in her organization, and she fills this position well';

- 'Elaine is a person who can manage her own affairs well'.

Il est évident que cette phrase appelle une traduction différente selon le contenu qu'on lui assigne, car si en anglais «manager» peut dénoter et le titre conféré à la personne occupant un certain poste dans la hiérarchie administrative, et le fait de s'acquitter des affaires de la vie courante, il n'existe pas en français de signifiant recouvrant ces deux signifiés. Autrement dit, l'homonyme anglais n'a pas pour correspondant un homonyme français. Inversement le transcodage de deux signifiants distincts dans la LD peut aboutir à un couple homonymique dans la LA : 
(5) A roast basted with a half bottle of red wine IUn rôti arrosé d'une demi-bouteille de vin rouge

[retraduction]

-... basted with ...

-... washed down with ...

L'exemple (5) illustre ce que nous entendons par l'ambiguïté interlinguistique.

L'ambiguïté syntaxique est tout aussi fréquente que l'ambiguïté lexicale, et généralement beaucoup plus difficile à lever. Voyons le cas de la coordination.

(6) J'aime le poulet et le chocolat

peut s'interpréter de deux façons, soit

- 'j'aime le poulet et $\mathrm{j}$ 'aime le chocolat'

- 'j'aime ceci : le poulet accompagné de chocolat'

La seconde interprétation est en effet tout aussi plausible que la première puisque la langue permet de dire «le pain et la confiture» où et signifie avec, et que par ailleurs le poulet au chocolat est un plat fort apprécié des Mexicains. L'ambiguité syntaxique vient de ce que la structure superficielle d'une phrase peut correspondre à des structures profondes différentes. La fidélité au TO suppose que toutes les ambiguiltés ont été levées au cours du décodage, qu'aucune n'a été introduite au moment du recodage, et que celles que l'auteur aurait pu glisser de propos délibéré dans son énoncé ont été conservées. Or, la difficulté en traduction tient moins aux problèmes posés par le recodage, encore que ceux-ci soient réels, qu'à la découverte des ambiguïtés au cours du décodage. Le lecteur procède en effet à une interprétation personnelle du texte qui a lieu de façon si spontanée qu'elle tend à éliminer toute autre interprétation possible. Il est donc souhaitable que le traducteur soit alerté et sensibilisé sur ce point. Un relevé exhaustif des ambiguités syntaxiques - plus insidieuses, semble-t-il, que les ambiguités lexicales - constitue sans doute une tâche impossible, mais les exemples qui suivent serviront à faire apprécier la nature et l'ampleur du problème.

Ambiguité catégorique :

Français

(7) Vous connaissez tous les effets de ces mesures tous $=$ adjectif $/$ nom

(8) Le psychanaliste parle au patient de sa voix enrouée $d e=$ préposition régime (vide)/préposition lexicale (pleine)

Anglais

(9) Kelpie was still next to the baby still $=$ adjectif $/$ adverbe

(10) Fruit flies like pineapple fies $=$ nom $/$ verbe like $=$ verbe $/$ conjonction

Ambiguïté fonctionnelle : Français

(11) Quels peintres ont imité les graveurs? 
peintres $=$ sujet $/$ objet

graveurs $=$ objet $/$ sujet

(12) J'ai oublié votre adresse à la maison

à la maison $=$ complément $\mathrm{du}$ verbe $/ \mathrm{du}$ nom

Anglais

(13) Who had Nick declared dead?

who $=$ sujet $/$ objet

(14) Man condemned to life in New York.

in New York = complément de phrase/complément de nom

Français

Clôture de phrase et de syntagme :

(15) Les Américains ont invité les Australiens et les Canadiens aussi et les Canadiens aussi = fin de la première phrase/début de la seconde phrase (et les Canadiens aussi ont invité les Australiens)

\section{Anglais}

(16) They went about stealthily glancing at the cakes in the window stealthily $=$ modifie went about $/$ modifie glancing

Comme nous l'avons déjà signalé, certaines ambiguïtés ne deviennent évidentes qu'au contact de deux langues. Le genre des adjectifs possessifs fait problème dans la traduction du français à l'anglais, puisque le français ne renseigne pas sur le sexe du possesseur, alors que l'anglais doit obligatoirement fournir cette indication. La conjugaison du verbe anglais comporte un mode progressif que le français ignore; par contre, le français dispose de nombreuses formes verbales pour exprimer le moment et l'aspect de l'action, tandis que le système des temps verbaux anglais est pauvre; en revanche, les modaux de l'anglais sont riches de formes et de sens, alors que le français n'a guère de véritables modaux. Et ainsi de suite : la liste est longue des asymétries entre les deux langues, asymétries qui engendrent souvent des ambiguités interlinguistiques. La phrase suivante, pourtant claire en apparence, illustre assez bien le genre de difficultés que nous évoquons :

(17) Son beau-père l'aide

$$
\begin{aligned}
& \text { son }=\text { his /her } \\
& \text { beau-père = father-in-law/stepfather } \\
& \text { l'=her/him } \\
& \text { aide = helps/is helping }
\end{aligned}
$$

Tant au point de vue du lexique que de la syntaxe, le sens des énoncés varie suivant les circonstances de l'énonciation, et l'on peut dire «Je ne vous aime pas» pour signifier exactement le contraire. Le choix de tel énoncé plutôt qu'un autre dans telle ou telle circonstance est évidemment dicté par des considérations personnelles, mais les conventions sociales imposent cependant au locuteur des habitudes discursives qui finissent par caractériser chaque groupe linguistique, qu'il s'agisse de sous-groupes (femmes, adolescents, ouvriers, sportifs, universitaires, partis politiques, et le reste) ou d'un grand groupe linguistique reconnu officiellement (anglophones, francophones, hispanophones, et ainsi de suite). Nous touchons là aux rapports entre la langue et la «culture ", 
entre le signifié dans la langue et le référent dans la réalité. Or, et c'est une source d'ambiguité fréquente, on n'emploie pas nécessairement les mêmes signifiés pour désigner des référents comparables selon que l'on utilise une langue ou une autre. À titre d'exemple, citons le cas de friend et ami qui s'appliquent à des personnes avec lesquelles on entretient des rapports présentant un degré d'intimité différent: deux signifiés équivalents, 'personne + lien + amitié' et 'person + bond + friendship' apparaissent dans ces circonstances différentes selon les groupes (Anglais, Américains, Canadiens, Français, Québécois, jeunes, vieux, conservateurs, "dans le vent»). Au niveau syntaxique, l'emploi de l'impératif semble évoquer un rapport d'autorité plus marqué aux États-Unis qu'en Angleterre et dans le monde francophone, d'où l'habitude d'en atténuer la portée en ajoutant please, et de lui substituer, en américain surtout, des tournures euphémiques, telles que la pseudo question would you (do thus and such) et la pseudo assertion I want you to (do such and such), comme dans les exemples suivants

(18) a Please come back at two

b Would you come back at two

c I want you to come back at two

= 'revenez à deux heures'

qu'il serait faux de traduire respectivement par

a' S'il-vous-plait, revenez à deux heures

b'Voulez-vous revenir à deux heures

c'Je veux que vous reveniez à deux heures

Le français a aussi ses euphémismes, comme l'imparfait et le conditionnel employés pour signifier un rapport de déférence à l'égard d'un supérieur.

(19) J'étais venu vous demander l'autorisation d'assister à votre conférence

ne signifie pas nécessairement que l'action s'est produite dans le passé. Et le vous de politesse ne permet pas toujours de savoir à combien d'interlocuteurs on s'adresse. Cela n'a pas d'importance lorsqu'on traduit vers l'anglais, puisque you y est également ambigu, mais peut faire problème lorsque la LD est l'espagnol.

Les euphémismes conventionnels, de même que les images figées (par exemple, en anglais you are pulling my leg ou encore, en français, la glace est rompue) semblent délimiter une interface entre le linguistique et le discursif. D'un côté, le sujet parlant est soumis à des contraintes qu'il ne peut récuser qu'en s'exposant au ridicule ou à l'incompréhension. De l'autre, il conserve sa liberté, celle de parler ou de se taire, celle d'être clair ou obscur, d'assumer la responsabilité de ses paroles ou de se dérober, de mettre une distance plus ou moins grande entre lui-même et le monde, d'établir un certain degré de tension vis-à-vis d'autrui, en d'autres termes, d'imprimer une marque personnelle à son énoncé. Il peut donc, s'il le veut, choisir de s'exprimer dans l'ambiguité. L'équivoque, le jeu de mots, les expressions figurées sont pour cela des moyens de prédilection. La publicité le sait, qui en fait grand usage.

(20) Take it ... easy 
Cette légende au-dessous d'une illustration représentant des appareils photographiques fait entendre deux messages simultanément,

- 'prenez-la [la photo], c'est facile'

- 'ne vous faites pas de souci'

en jouant sur les mots. Comme take it easy sert actuellement de formule pour prendre congé entre amis, le texte publicitaire donne à comprendre indirectement que le temps est venu de partir en vacances ou en voyage muni d'un appareil facile à manier et offert par un fabricant qui ne vous veut que du bien, comme un ami. Très astucieux ... et intraduisible (au sens strict du terme).

Les mots sans référence jouent également le jeu de l'ambiguïté. Le message qui suit ne semble pas perdre en intensité ce qu'il gagne en extension :

(21) Do it now!

Cet ordre peut en effet s'appliquer à tout, donc à rien en particulier, puisque do et it sont des éléments de substitution, et que dans un énoncé isolé, ils ne remplacent ni un verbe ni un nom ou une phrase dotés d'un contenu identifiable.

Enfin, les phrases absurdes et les phrases contradictoires rendent aussi des services à l'ambiguïté. Que signifie exactement ce slogan publicitaire?

(21) One of the largest banks in the world is taking a new path to intercept the future (Fortune July 14th, 1980).

Le contexte qui l'accompagne n'est guère plus éclairant. Sans doute chacun est-il libre de l'entendre selon ses désirs, ses craintes et ses aspirations, ce qui est sûrement ce que souhaitait son auteur. Traducteur, en garde!

Les remarques sur l'ambiguité qui précèdent ont surtout valeur d'avertissement. Elles nous ont été inspirées par notre travail de professeur, certes, mais encore davantage par les travaux de révision qu'il nous arrive de faire à l'occasion, ainsi que par la lecture de certaines traductions dûment éditées. Nous aimons à croire qu'elles ne sont pas totalement dépourvues d'intérêt. 